\title{
EXPECTATIVAS SOBRE LA XXI CONFERENCIA DE LAS PARTES
}

\section{EXPECTATIONS ON THE XXI CONFERENCE OF THE PARTIES}

\author{
Rogelio Nicolás Macines Romero* \\ Docente Asociado de la Facultad de Ciencias Económicas \\ Universidad Nacional Mayor de San Marcos - UNMSM / Lima - Perú \\ [Recepción: Setiembre de 2015 / Conformidad: Octubre de 2015]
}

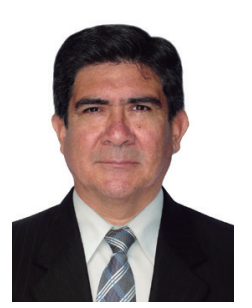

\section{RESUMEN}

Uno de los grandes problemas globales que está afectando a la sociedad y; particularmente, a los bajos estratos sociales es el relacionado al medioambiente y al cambio climático. Es una realidad la pérdida de hielo en los polos terráqueos y en los glaciares de las montañas como ocurre en nuestro país, los fenómenos climáticos cada vez son más intensos como los huracanes, las tormentas, los tifones, las sequías, el Fenómeno del Niño.

A pesar de existir mayor consenso sobre los graves daños potenciales, los países por diversos motivos aún no han encontrado la forma de continuar con el camino señalado por el Protocolo de Kioto, teniendo una nueva oportunidad en la XXI Conferencia de las Partes, que se llevará a cabo en diciembre de 2015 en París.

En este artículo, analizamos dos de las razones que dificultan a los países ponerse de acuerdo como son: 1. El nivel de la actividad económica de los países expresadas en sus tasas de crecimiento. Si este crecimiento es bajo el problema del cambio climático queda relegado y 2. El petróleo considerado el principal recurso energético que se utiliza a nivel mundial y también es una de las principales fuentes de contaminación del medioambiente.

\section{Palabras clave:}

Conferencia de las partes; cambio climático; petróleo.

\section{ABSTRACT}

One of the major global problems that is affecting society and particularly the lower social strata is related to the environment and climate change. The earth poles are losing ice as well as glaciers in our country are melting, weather events are becoming more intense: hurricanes, storms, typhoons, droughts, El Niño.

Although there is greater consensus on the potential serious damage, countries for various reasons have not yet found a way to continue the path indicated by the Kyoto Protocol, taking a new opportunity in the XXI Conference of the Parties in December 2015 in Paris. In this article we analyze two of the reasons that make difficult for countries to agree: 1 . The level of economic activity of the countries is expressed on their rates of growth. If this growth is low, the issue of climate change is relegated; 2 . The oil is considered to be the main source of energy used worldwide and it is also the major source of environmental pollution.

\section{Keywords:}

Conference of the parties; climate change; oil.

* Magíster en Economía con mención en Finanzas. Email: rmacines@yahoo.com 


\section{INTRODUCCIÓN}

Las Naciones Unidas se van a reunir en diciembre de 2015 en París, para negociar un acuerdo que permita enfrentar uno de los problemas críticos que está afectando a nuestra sociedad como es el cambio climático, es la reunión anual número XXI y existen muchas expectativas para un final positivo.

En la reunión número XX de Lima, se logró un avance de esta negociación entre los diferentes puntos de vista sobre el cambio climático, llegándose a acordar un documento al que se denominó Borrador, donde se incorporaron los temas y algunos lineamientos a discutirse en París.

Pero el análisis que desarrollamos en este artículo precisamente se enfoca en dos aspectos que limitarían esas expectativas; primero, señalamos la incidencia negativa de los bajos niveles de crecimiento a nivel global y segundo, señalamos a los bajos precios del petróleo que incentivan su uso y por lo tanto, tiende a incrementarse los gases contaminantes.

\section{LA ACTIVIDAD ECONÓMICA Y LAS NEGOCIACIONES SOBRE EL CAMBIO CLIMÁTICO}

Desde la década de los 80 del siglo pasado, el cambio climático se ha convertido en otro de los graves problemas globales que enfrentan las Naciones Unidas, debido a las concentraciones de gases en la atmósfera que están incrementando el efecto invernadero y sobre el cual, se realizan reuniones anuales tratando de encontrar una solución que satisfaga a todas las naciones, es un consenso científico que la demora en los acuerdos para reducir la emisión de los gases de efecto invernadero causará graves daños sobre el planeta, incrementándose las sequías por un lado, inundaciones por otro, mayor intensidad de tormentas, tornados, Fenómeno del Niño, olas de calor, extinción de algunas especies, pérdida de glaciares en las montañas, poniendo en riesgo el abastecimiento de agua, aparición de pandemias, pérdida de infraestructuras, mayor riesgo en el abastecimiento de alimentos, etc.

A nuestro entender, dos de los factores que influyen en las negociaciones sobre el cambio climático es el nivel del crecimiento económico de los países y el petróleo como principal recurso energético utilizado.

La actividad económica se caracteriza por presentar ciclos de auge y crisis, que en la actualidad se extienden rápidamente por todo el planeta debido a la globalización, pero a pesar de los períodos de auge en el aspecto social aún persisten problemas como son los altos niveles de pobreza, la discriminación, la salud, el acceso a servicios básicos, la desigualdad, etc.

En la década de los 90, el Consenso de Washington impulsado por el Fondo Monetario Internacional y el Banco Mundial significó para la mayoría de los países en desarrollo la adopción y creencia que el modelo basado en el mercado con la liberalización de las inversiones y el comercio, privatización de empresas públicas, mayor disciplina fiscal, etc., les permitiría alcanzar el anhelado nivel de desarrollo logrado por las economías avanzadas, pero como lo demuestran los datos (Ver cuadro No 01) precisamente son estas economías quienes han ido disminuyendo sus niveles de crecimiento con el mismo modelo económico.

Las economías avanzadas en la década de los 80 habían alcanzado niveles de crecimiento con un promedio anual de $3.57 \%$ y en los 90 este promedio llegó a decaer a $3.32 \%$, y en la primera década del siglo XXI el crecimiento de las economías avanzadas, siguió cayendo hasta llegar a un promedio anual de $2.60 \%$, agravando la situación el estallido de la crisis financiera en el 2008, que según algunos analistas se origina por la falta de una debida regulación

\section{Cuadro No 01}

Tasa de crecimiento promedio del PBI (anual)

\begin{tabular}{|l|c|c|c|c|}
\hline & $\mathbf{1 9 8 0 - 1 9 8 9}$ & $\mathbf{1 9 9 0 - 1 9 9 9}$ & $\mathbf{2 0 0 0 - 2 0 0 9}$ & $\mathbf{2 0 1 0 - 2 0 1 4}$ \\
\hline Economías avanzadas & 3.57 & 3.32 & 2.61 & 1.47 \\
\hline Economías emergentes y en desarrollo & 2.95 & 3.70 & 6.03 & 4.08 \\
\hline
\end{tabular}

Fuente: Datos del FMI: World Economic Outlook. Elaboración propia. 
sobre el mercado de derivados y en general, sobre el mercado financiero, pero que otros señalan como verdadera causa la inconsistencia que existe entre el crecimiento del sector productivo y el crecimiento del sector financiero. En la presente década las economías avanzadas siguen presentando bajas tasas de crecimiento que llegan al $1.47 \%$ en promedio anual.

En comparación, las tasas de crecimiento promedio anual de las economías emergentes y en desarrollo, para los mismos períodos fueron: $2.95 \%$, $3.70 \%, 6.03 \%$ y $4.07 \%$ respectivamente, existiendo repercusión de la crisis en estas economías en la presente década, pero a pesar de ello, la Conferencia de las Naciones Unidas sobre Comercio y Desarrollo (UNCTAD, por sus siglas en inglés) señala el aumento de la participación de las economías en desarrollo en el PBI mundial pasando del 17\% en 1990 al 36\% en el 2012. (UNCTAD, 2013: p. 12).

En este escenario cuando se empieza a discutir el problema del cambio climático coincidentemente en los 80 y 90, la mayoría de países desarrollados se encontraban con mayor capacidad y estabilidad económica para enfrentarlo, por lo que existía mayor aceptación de los informes sobre el cambio climático, pero ya en la década de los 90 no se lograron los niveles de crecimiento de la década anterior, peor desempeño tuvieron en la década del 2000, reflejándose a nuestro entender en las negociaciones sobre el cambio climático, por ejemplo el Protocolo de Kioto que fue vinculante para las economías avanzadas y aprobado en 1997 entró en vigencia recién en el 2005 al cumplirse la condición que lo ratificasen los países industrializados responsables de al menos el $55 \%$ de las emisiones de $\mathrm{CO} 2$ y cuyo cumplimiento sería en el período 2008-2012 (Mendiola et al, 2008, p. 38); EE.UU. no ratificó el Protocolo por lo cual no estaba obligado a él.

Por el contrario, las economías emergentes y en desarrollo habían elevado su desempeño económico en la década de los 90 y aún más, en la década del 2000. Es por ello, que las economías avanzadas están exigiendo que estos países acepten un mayor compromiso en las negociaciones climáticas a pesar de que la acumulación existente de los gases de efecto invernadero es mayormente responsabilidad de los países desarrollados; pero debido a esas altas tasas de crecimiento, algunos países podrían haberse convertido en emisores importantes de estos gases, como lo es el caso de China que durante muchos años lidera los niveles de crecimiento.

China, también representa el anhelo de los países emergentes y en desarrollo, lograr un constante elevado nivel de crecimiento que permita mejorar el bienestar de su habitantes con la oportunidad de reducir las brechas sociales, elevando los niveles de educación, salud, servicios básicos, infraestructuras, etcétera, pero al igual que ella, también poseen el mismo problema de tener tecnologías atrasadas e ineficientes para sostener ese crecimiento, estas tecnologías son altamente contaminantes por lo cual se relega el aspecto medioambiental a pesar de implementarse políticas internas que terminan siendo débiles al no ser parte de un acuerdo internacional.El cambio climático es un problema global que requiere un acuerdo global.

\section{EL PETRÓlEO Y EL CAMBIO CLIMÁTICO}

Los recursos naturales renovables y no renovables son importantes y necesarios para la actividad económica de los países, por ejemplo: los bosques, biomasa marina, el agua, los minerales, los hidrocarburos, etc. El petróleo es considerado el principal recurso energético no renovable utilizado en toda actividad económica desde el comienzo de su explotación en el siglo XIX siendo uno de los factores que favoreció la Revolución Industrial, etapa que los científicos consideran se inicia el problema del cambio climático.

En los años 80 se constituye el Panel Intergubernamental sobre el Cambio Climático (IPCC por las siglas en inglés) con científicos de todo el mundo, para revisar los informes que se estaban publicando referidos a los cambios que estaban ocurriendo en la atmósfera y evaluar sus causas y consecuencias. Este organismo llegó a concluir que el origen de estos cambios era el incremento del efecto invernadero; si bien existe, un efecto invernadero natural que protege a nuestro planeta haciendo posible que haya vida a temperaturas aceptables, el Panel sostiene que la actividad antropogénica ha contribuido a elevar la emisión de diversos gases que se han estado acumu- 
lando en la atmósfera elevando el efecto invernadero. El dióxido de carbono (CO2) es uno de estos gases $y$ es considerado el de mayor volumen acumulado en la atmósfera, el petróleo es uno de los principales emisores de este gas.

Hasta comienzos de la década de los 70 del siglo pasado, el precio por barril era relativamente bajo no existiendo otro recurso energético que lo sustituya en esas dimensiones, fue en 1973 cuando ocurrió el aumento de su precio que supera el $200 \%$ originándose la llamada primera crisis energética más su incremento sucesivo (Ver cuadro No 02) es que se obliga a las economías avanzadas a investigar e invertir en proyectos de alternativas energéticas tratando de reducir su dependencia.

El precio promedio por barril de petróleo alcanzó los 36.83 dólares en 1980 manteniéndose por debajo de ese precio en las dos décadas siguientes alcanzando su más bajo valor en 1998 que fue 12.72 dólares; pero a partir de ese año, empezó un aumento que no se detuvo hasta el año 2012 superando la barrera de los
100 dólares por barril, llegando a un precio promedio anual de 111.67 dólares.

Actualmente, la tecnología de explotación del petróleo se ha modernizado reduciendo costos de tal manera que en el presente año 2015 su precio ha caído nuevamentes por debajo de los 50 dólares por barril, pero continúa siendo el principal recurso energético para la actividad económica por lo que nuevamente existe incertidumbre sobre los proyectos alternativos de energía más limpia y sostenible.

La sociedad es altamente dependiente del petróleo como recurso energético, por lo que para los procesos productivos solo se toma en cuenta el costo por barril en el mercado, no considerando el costo de la externalidad negativa que ocasiona su uso. Es decir, la emisión de $\mathrm{CO} 2$ que no es absorbida por el planeta se sigue acumulando en la atmósfera incrementando el efecto invernadero y originando los cambios señalados líneas arriba, por lo que el costo social de su uso es mucho más alto.

\section{Cuadro No 02}

Precio promedio del petróleo

\begin{tabular}{|c|c|c|c|c|c|}
\hline Año & $\begin{array}{c}\text { U.S.\$ / } \\
\text { Barril }\end{array}$ & Año & $\begin{array}{c}\text { U.S.\$ / } \\
\text { Barril }\end{array}$ & Año & $\begin{array}{c}\text { U.S.\$ / } \\
\text { Barril }\end{array}$ \\
\hline 1973 & 3.29 & 1987 & 18.44 & 2001 & 24.44 \\
\hline 1974 & 11.58 & 1988 & 14.92 & 2002 & 25.02 \\
\hline 1975 & 11.53 & 1989 & 18.23 & 2003 & 28.83 \\
\hline 1976 & 12.80 & 1990 & 23.73 & 2004 & 38.27 \\
\hline 1977 & 13.92 & 1991 & 20.00 & 2005 & 54.52 \\
\hline 1978 & 14.02 & 1992 & 19.32 & 2006 & 65.14 \\
\hline 1979 & 31.61 & 1993 & 16.97 & 2007 & 72.39 \\
\hline 1980 & 36.83 & 1994 & 15.82 & 2008 & 97.26 \\
\hline 1981 & 35.93 & 1995 & 17.02 & 2009 & 61.67 \\
\hline 1982 & 32.97 & 1996 & 20.67 & 2010 & 79.50 \\
\hline 1983 & 29.55 & 1997 & 19.09 & 2011 & 111.26 \\
\hline 1984 & 28.78 & 1998 & 12.72 & 2012 & 111.67 \\
\hline 1985 & 27.56 & 1999 & 17.97 & 2013 & 108.66 \\
\hline 1986 & 14.43 & 2000 & 28.50 & 2014 & 98.95 \\
\hline
\end{tabular}

De 1973 a 1983: Precio de Petróleo ARABE ligero

De 1984 a 2013: Precio de Petróleo BRENT

Fuente: Statistical Review of World Energy 2014. Tomado el dia 19-4-15

(http://www.bp.com/en/global/corporate/about-bp/energy-economics/statistical-review-of-world- energy.htm) 


\section{DE LA COP 20 DE LIMA A LA COP 21 DE PARÍS}

En el marco de las Naciones Unidas sobre el cambio climático, las Conferencias de las Partes se vienen reuniendo anualmente desde 1995, siendo la más relevante la del año 1997, cuando se elaboró el Protocolo de Kioto con el compromiso por parte de los países desarrollados (con excepción de EE. UU.) de reducir un $5.2 \%$ las emisiones de gases de efecto invernadero medidos en el año 1990, estableciendo como plazo del 2008 al 2012.

Después de ese compromiso los países no han logrado ponerse de acuerdo para enfrentar el cambio climático y para evitar prolongar más estas reuniones, se ha establecido el año 2015 la fecha límite para adoptar un acuerdo en París que entrará en vigencia el 2020. En diciembre de 2014 se realizó la Conferencia de las Partes número 20 (COP 20) en Lima con el objetivo de elaborar el Borrador del acuerdo a discutirse en la COP 21 ; y como recordamos hubo que prolongarse la última sesión después de la medianoche, para lograr este documento cuyos objetivos a muchos no convenció, existiendo incertidumbre sobre los logros a conseguir en la reunión de París.

En las negociaciones sobre el cambio climático existen bloques de países para defender intereses comunes, por ejemplo los grupos regionales: África, Asia, Europa central y oriental, Latinoamérica y el Caribe, y Europa occidental junto a Australia, Canadá, USA, Fernando Tudela (2014) de CEPAL señala:

"En la actual etapa de las negociaciones, tal vez el principal problema de fondo radica en el creciente desfase entre el alcance de las acciones y compromisos actuales, o incluso el de los que pudieran a corto plazo ser objeto de un acuerdo multilateral, y aquellos que serían necesarios para controlar el cambio climático al imponer límites de emisión consistentes con el objetivo global declarado de mantener el aumento de la temperatura promedio por debajo de los $2^{\circ} \mathrm{C}$. En otras palabras, o bien se aumenta el nivel de acciones y compromisos, asegurando su cumplimiento, o bien se resignan los países a sobrevivir en un mundo cuyo clima habrá sido transformado más allá de la voluntad expresa de sus dirigentes.” p. 51
Tal vez exista consenso en la COP 21 y se logre un acuerdo para enfrentar el cambio climático de manera conjunta, pero debido a la acumulación de los gases existentes y aún si se reducen las emisiones a cero, se espera que el cambio climático y sus efectos negativos continúen por un tiempo. De tal manera, que el siglo XXI podría ser reconocido más adelante como el siglo de los efectos del cambio climático, es decir, profundos cambios en ecosistemas, agricultura, pesca, salud, fenómenos atmosféricos intensos, etcétera, por lo que estamos advertidos y debemos prepararnos y preparar a las futuras generaciones para un nuevo escenario ambiental que no han ocurrido anteriormente.

Del mismo modo, debemos cambiar nuestra actitud en relación al cambio climático, lo que implica un cambio a nivel de consumidores, de los empresarios y de los gobernantes, para lo cual se necesita un proceso permanente de concientización de la sociedad.

\section{CONCLUSIONES}

1. Existe la necesidad de que los países adopten un acuerdo para enfrentar el cambio climático que deberá contemplar el cambio del modelo económico basado en la economía de mercado, porque presenta limitaciones para resolver los problemas de la sociedad y por el contrario afecta el medioambiente creando nuevos problemas.

2. En la actualidad, no hay un real compromiso de cambio de actitud de consumidores, empresarios y gobernantes, por ejemplo en la construcción de infraestructuras (puentes, carreteras, hidroeléctricas, etc.) donde deberá tomarse en cuenta el escenario futuro como consecuencia del cambio climático, seguir haciéndolo como hasta ahora significará cometer los mismos errores que impliquen altos costos económicos y sociales.

3. La sociedad actual aún es altamente dependiente del petróleo como el principal recurso energético, y también uno de los importantes emisores de gases que se están acumulando en la atmósfera que va incrementando el efecto invernadero, por lo que los productores y consumidores de este re- 
curso tienen la oportunidad en las negociaciones de París para establecer escenarios energéticos futuros que reduzca considerablemente los riesgos climáticos.

4. Existe el riesgo que en la Conferencia de las Partes a realizarse este año, se apruebe un documento con objetivos reales para enfrentar el cambio climático y vinculante para los países que lo ratifiquen, pero que no entrará en vigencia mientras no lo ratifiquen las principales economías sean desarrolladas, emergentes o en desarrollo. Asimismo, siempre estará latente la posibilidad de una crisis económica internacional que hará difícil cumplir con dichos compromisos.

\section{REFERENCIAS BIBLIOGRÁFICAS}

1. BRITISH PETROLEUM (2015) Statistical Review of World Energy 2014, versión electrónica, recuperado de http://www.bp.com/en/global/ corporate/about-bp/energy-economics/ statistical-review-of-world-energy.html

2. ESCRIBANO, J. (2013) Cambio climático, política, economía y derecho: Desafíos para la Comunidad Internacional, versión electrónica, recuperado de http://e-archivo.uc3m.es/ bitstream/handle/10016/17219/monografias_3_ IFV_2013.pdf?sequence $=1$
3. FONDO MONETARIO INTERNACIONAL (2014) World Economic Outlook, versión electrónica, recuperado de https://www.imf.org/ external/pubs/ft/weo/2014/02/weodata/index. aspx

4. IPCC (2014) Cambio climático 2014, versión electrónica, recuperado de https://www.ipcc. $\mathrm{ch} / \mathrm{pdf} /$ assessment-report/ar5/wg2/ar5_wgII_ spm_es.pdf

5. MENDIOLA, A., FUENTES, C., ARROYO, W., AUQUI, M., HUAMAN, R., MARTINEZ,J., (2008) El desarrollo del mercado de carbono en el Perú, Ed. Esan.

6. TUDELA, F. (2014) Negociaciones internacionales sobre el cambio climático. Ed. CEPAL, Documento de proyecto, versión electrónica, recuperado el 26-4-15 de http://repositorio.cepal.org/ bitstream/handle/11362/37329/S1420809 es.pdf;jsessionid $=0 \mathrm{BC} 7 \mathrm{EC} 487 \mathrm{C08709ACABEB}$ 1853036DD91 ?sequence $=1$

7. UNCTAD (Conferencia de las Naciones Unidas sobre comercio y desarrollo), (2013) Informe sobre el comercio y el desarrollo, 2013 Documento UNCTAD/TDR/2013, versión electrónica en: http://unctad.org/es/PublicationsLibrary/ tdr2013overview_es.pdf 